\title{
Gentamicin B1 is not a minor gentamicin component with major nonsense mutation
}

\section{suppression activity}

Alireza Baradaran-Heravi ${ }^{1}$, David E. Williams ${ }^{2}$, David A. Powell ${ }^{3}$, Aruna D. Balgi ${ }^{1}$, Raymond J. Andersen $^{2}$ and Michel Roberge ${ }^{1 *}$

${ }^{1}$ Department of Biochemistry and Molecular Biology, University of British Columbia, Vancouver, British Columbia, Canada, V6T 1Z3

${ }^{2}$ Department of Chemistry, University of British Columbia, and Department of Earth, Ocean \& Atmospheric Sciences, University of British Columbia, Vancouver, British Columbia, Canada, V6T 1 Z4

${ }^{3}$ Inception Sciences, Vancouver, British Columbia, Canada, V5T 4T5

${ }^{*}$ Corresponding Author: Michel Roberge, Department of Biochemistry and Molecular Biology, University of British Columbia, 2350 Health Sciences Mall, Vancouver, British Columbia, Canada, V6T 1Z3. Tel. (604) 822-2304. Email: michelr@mail.ubc.ca

Key words: Gentamicin B1, G418, nonsense mutation, premature stop codon readthrough 


\begin{abstract}
Nonsense mutations are single base substitutions that introduce a premature termination codon (PTC) preventing the formation of full-length protein. They are the causative mutations in about $10 \%$ of patients in a large number of rare genetic diseases. High concentrations of the antibiotic gentamicin can induce the incorporation of an amino acid at a PTC and formation of full-length protein, a process called PTC readthrough. Gentamicin is composed of several related aminoglycosides. We recently reported (doi/10.1073/pnas.1620982114) that the major gentamicin components that are responsible for its antibacterial activity showed weak to no PTC readthrough activity but that the minor component gentamicin B1 was a potent readthrough inducer. We have now determined that gentamicin B1 acquired from the sole supplier at the time the study was carried out was not gentamicin B1 but instead the closely related aminoglycoside G418. Gentamicin B1 recently became available from a second commercial source. Here, we provide nuclear magnetic resonance (NMR) assignment data for the two commercial compounds and verify only the second is indeed gentamicin B1. We show that gentamicin B1 lacks PTC readthrough activity in HDQ-P1 and DMS-114 cells homozygous for the TP53 R213X nonsense mutation, as well as in a cell-free translation assay.
\end{abstract}




\section{Introduction}

Nonsense mutations in the coding sequence of a gene change an amino acid codon to a premature termination codon (PTC) and are responsible for about $10 \%$ of rare genetic disease cases (1). Compounds that permit insertion of an amino acid at the PTC enable formation of full-length protein and increased protein function, instead of truncated inactive protein. This strategy, termed nonsense suppression or PTC readthrough, has the potential to treat large numbers of patients across multiple rare genetic disorders. However, drugs that can induce therapeutic levels of readthrough at safe doses are not yet available.

Gentamicin is an aminoglycoside antibiotic approved for use in humans. It can induce PTC readthrough in cultured cells but only at concentrations that are orders of magnitude higher than the plasma concentration considered safe in humans (2). Clinical trials in Duchenne muscular dystrophy and cystic fibrosis patients have indicated that therapeutically relevant levels of readthrough cannot be achieved at subtoxic gentamicin doses (3-5). Gentamicin is not a pure compound but a mixture of related aminoglycosides isolated from the soil fungus

Micromonospora purpurea. It is composed of specified proportions of the major gentamicins $\mathrm{C} 1$, C1a, C2, C2a and C2b. The U.S. Pharmacopeial Convention (USP) requires $25-50 \% \mathrm{C} 1 ; 10-35 \%$ C1a; 25-55\% C2 + C2a while the European Pharmacopea specifies 20-40\% C1, 10-30\% C1a, 40$60 \% \mathrm{C} 2+\mathrm{C} 2 \mathrm{a}+\mathrm{C} 2 \mathrm{~b}(6)$. Gentamicin may also contain small amounts of a number of related aminoglycosides found in variable proportions in different drug batches and accounting together for $<3 \%$ of the material (6). Variable response to gentamicin treatment has been observed in animal models and humans $(5,7)$ and it has been speculated that this variability may be due to differences in gentamicin composition (7).

In a 2017 publication, we examined this question by measuring the activity of pure samples of 
each individual major gentamicin component and of minor gentamicin components (8). We found that none of the major gentamicins showed significant activity, an observation recently

corroborated by others (9). We reported that the minor component gentamicin B1 was 30- to 100fold more potent than the most potent gentamicin batches tested (8). In recent structure-activity relationship studies to be reported elsewhere, we observed that synthetic aminoglycoside analogs bearing ring 1 of gentamicin B1 lacked readthrough activity whereas analogs bearing ring 1 of the closely related aminoglycoside G418 showed readthrough activity. This puzzling observation led us to reexamine the structure and PTC readthrough activity of gentamicin B1.

\section{Materials and Methods}

NMR. All NMR spectra were recorded on a Bruker AV-600 spectrometer with a 5 mm CPTCI cryoprobe using standard Bruker pulse sequences. ${ }^{1} \mathrm{H}$ chemical shifts are referenced to the residual DMSO- $d_{6}(\delta 2.49 \mathrm{ppm})$ and ${ }^{13} \mathrm{C}$ chemical shifts are referenced to the DMSO- $d_{6}$ solvent peak ( $\delta 39.5 \mathrm{ppm})$.

Human cells. The HDQ-P1 cell line was purchased from the German Collection of Microorganisms and Cell Cultures (DSMZ). DMS-114 cells were purchased from the American Type Culture Collection (ATCC). Both have homozygous nonsense mutation in the TP53 gene (NM_000546.5:c.637C > T; NP_000537.3:p.R213X). HDQ-P1 and DMS-114 cells were cultured in high glucose Dulbecco's modified Eagle medium (DMEM, Sigma-Aldrich) and RPMI-1640 (Sigma-Aldrich), respectively, supplemented with 10\% (vol/vol) FBS (Sigma-Aldrich) and 1× antibiotic-antimycotic (Gibco/Thermo Fisher Scientific) at $37{ }^{\circ} \mathrm{C}$ and $5 \%$ (vol/vol) $\mathrm{CO}_{2}$.

Automated Capillary Electrophoresis Western Analysis. Detection of p53 was performed as previously described (10). Briefly, HDQ-P1 or DMS-114 cells were seeded in 12-well culture plates and incubated with various concentrations of compounds. After $48-72 \mathrm{~h}$ incubation the 
cells were washed with ice-cold PBS and lysed in $50 \mu$ lysis buffer composed of $20 \mathrm{mM}$ Tris $\cdot \mathrm{HCl}, \mathrm{pH}$ 7.5, $150 \mathrm{mM} \mathrm{NaCl}, 1 \mathrm{mM}$ EDTA, 1 mM EGTA, 1\% (vol/vol) Triton X-100, 2.5 $\mathrm{mM}$ sodium pyrophosphate, $1 \mathrm{mM} \beta$-glycerophosphate, $1 \mathrm{mM} \mathrm{Na}_{3} \mathrm{VO}_{4}, 1 \mathrm{mM}$ DTT, and $1 \times$ complete protease inhibitor mixture (Roche Molecular Biochemicals). Lysates were centrifuged at $18,000 \mathrm{~g}$ for $15 \mathrm{~min}$ at $4{ }^{\circ} \mathrm{C}$ and protein level was quantitated in the supernatants using the Bradford assay and adjusted to 0.9 or $0.7 \mathrm{mg} / \mathrm{ml}$ protein for HDQ-P1 and DMS-114 cells, respectively. Capillary electrophoresis western analysis was carried out following the manufacturer's instructions (ProteinSimple WES) using DO-1 p53 antibody from Santa Cruz Biotechnology and vinculin antibody (clone 728526) from R\&D Systems. The data were analyzed with the inbuilt Compass software (ProteinSimple).

Aminoglycosides. G418 disulfate was purchased from Sigma (A1720). Gentamicin B1 (B1MCC) isolated from gentamicin sulphate complex $\mathrm{C}$ was purchased from MicroCombiChem, Germany as free base and as sulfate salt. Synthetic gentamicin B1 acetate (B1-TRC) was purchased from Toronto Research Chemicals.

In Vitro Transcription and Translation Assays. In vitro transcription and translation assays were performed as previously described (10) with minor modifications. In brief, pcDNA-6.2/V5DEST vector expressing p53 R213X-TGA was linearized with FastDigest MssI restriction enzyme (Thermo Fisher Scientific) and subjected to RNA synthesis using the mMESSAGE mMACHINE T7 ULTRA Transcription Kit (Thermo Fisher Scientific). 500 ng of the 5' capped and poly(A)- tailed RNA was subjected to in vitro translation using the One-Step Human Coupled IVT Kit (Thermo Fisher Scientific). This assay was carried out at $30^{\circ} \mathrm{C}$ for $30 \mathrm{~min}$ in the presence or absence of various concentrations of gentamicin B1 or G418 according to the manufacturer's instructions. Samples were diluted 10 times in water and $5 \mu$ of each sample was subjected to automated capillary electrophoresis western analysis for p53 detection. 


\section{Results}

The commercial gentamicin B1 samples used in our 2017 study (8), here termed B1-MCC, were purchased as free base and as sulfate salt. They were provided with Certificates of Analysis, mass spectrometry and NMR data (Supplementary Figure 1) supporting designation as gentamicin B1, which was corroborated by an independent NMR analysis carried out at our request. During the summer of 2018, while carrying out structure-activity relationship studies of synthetic pseudotrisaccharides, we noticed that replacing ring 1 of G418 with ring 1 of gentamicin B1 caused loss of PTC readthrough activity (unpublished). This observation made us suspect the identity of B1-MCC.

Gentamicin B1 and G418 are closely related compounds with the same mass and molecular formula (496.55 g/mol; $\mathrm{C}_{20} \mathrm{H}_{40} \mathrm{~N}_{4} \mathrm{O}_{10}$ ) that differ only in the positions of the $\mathrm{NH}_{2}$ and $\mathrm{OH}$ functional groups at C-2' and C-6' as shown in the structural drawings in Figure 1. We have been unable to find any published reference NMR assignments for gentamicin B1 in the peer-reviewed or patent literature. The Certificate of Analysis provided by the vendor included ${ }^{1} \mathrm{H}$ and ${ }^{13} \mathrm{C} \mathrm{NMR}$ assignments for spectra recorded in $\mathrm{D}_{2} \mathrm{O}$ (Supplementary Figure 1). A resonance at $\delta 54.7$ was assigned to a secondary alcohol carbon $\mathrm{C}-2^{\prime}$ and a resonance at $\delta 65.6$ to an amine-bearing carbon C-6'. Support for the placement of the alcohol at C-2' and the amine at C-6' in this sample came from an ${ }^{1} \mathrm{H}_{-}{ }^{15} \mathrm{~N}-\mathrm{HMBC}$ spectrum recorded in $\mathrm{D}_{2} \mathrm{O}$ at the Centre for Drug Research and Development in Vancouver. This spectrum showed a strong ${ }^{1} \mathrm{H} /{ }^{15} \mathrm{~N}$ correlation that had a ${ }^{1} \mathrm{H}$ chemical shift of $\delta 1.13 \mathrm{ppm}$. A methyl doublet at $\delta 1.13$ in the $1 \mathrm{D}{ }^{1} \mathrm{H}$ NMR spectrum of this B1MCC sample could be confidently assigned to C-7' since it was the only methyl group in the molecule vicinal to a methine. They concluded that the strong ${ }^{1} \mathrm{H}_{-}{ }^{15} \mathrm{~N}-\mathrm{HMBC}$ correlation between Me-7' and a nitrogen was only possible if there was an amino functionality at C-6', suggesting that the sample was gentamicin B1. 
In summer 2018, synthetic gentamicin B1, here termed B1-TRC, became available as the acetate salt from a second commercial vendor. The Certificate of Analysis and vendor NMR data are shown in Supplementary Figure 2. We have carried out detailed 1D and 2D NMR analysis of both the B1-MCC and B1-TRC samples and our ${ }^{1} \mathrm{H}$ and ${ }^{13} \mathrm{C}$ assignments for both samples are listed in Table 1. Of particular note are our assignments of $\delta 56.2$ for C-2' and of $\delta 66.2$ for C- $6^{\prime}$ in the ${ }^{13} \mathrm{C}$ NMR spectrum of the B1-MCC sample recorded in DMSO- $d_{6}$. These values are in good agreement with the assignments of the ${ }^{13} \mathrm{C}$ shifts of these two carbons provided by the vendor for spectra recorded in $\mathrm{D}_{2} \mathrm{O}$ [ $\delta 54.7\left(\mathrm{C}-2^{\prime}\right)$; $\left.65.6\left(\mathrm{C}-6^{\prime}\right)\right]$ (Supplementary Figure 1) allowing for minor shifts due to different solvents. However, the C-2' resonance was more shielded and the C-6' was more deshielded than expected/calculated for the proposed gentamicin B1 structure that has an alcohol at $\mathrm{C}-2^{\prime}$ and an amine at $\mathrm{C}^{\prime}$. This suggested that the structure assigned by the vendor was incorrect and that the positions of the amino and alcohol functionalities needed to be switched, so that B1-MCC was actually G418. We then mixed B1-MCC with authentic G418 (from Sigma) and recorded $1 \mathrm{D}{ }^{1} \mathrm{H}$ NMR data on the mixture. This experiment showed that the two compounds were identical.

Our independent NMR structural analysis of the B1-TRC sample showed it is indeed gentamicin B1 (Table 1) and C-2' bearing an alcohol was assigned to a carbon resonance at $\delta$ 72.4 and C-6' now bearing an amino nitrogen was assigned to a carbon resonance at $\delta 47.8$. Hence, the key to distinguishing between the two structures G418 and gentamicin B1 is the correct assignment of the carbon chemical shifts of C-6' and C-2' to carbons bearing either amino or alcohol functionalities. The configuration at C-6' of B1-TRC remains undefined as it is not possible to assign this from the NMR analysis alone. Finally, it appears that the putative diagnostic ${ }^{1} \mathrm{H}^{15} \mathrm{~N}-\mathrm{HMBC}$ correlation observed at the Centre for Drug Research and Development arises from a correlation between $\mathrm{H}-2_{\mathrm{ax}}$ and one of the adjacent nitrogen atoms at either $\mathrm{C}-1$ or $\mathrm{C}$ - 
3. The $\mathrm{H}-2_{\mathrm{ax}}{ }^{1} \mathrm{H}$ resonance was presumably isochronous with the Me- ${ }^{\prime}{ }^{1} \mathrm{H}$ resonance since we also observed nearly identical chemical shifts for Me-7' $(\delta 1.01)$ and $H-2_{a x}(\delta 0.99)$ in our data for B1-MCC (Table 1).

We compared the PTC readthrough activity of B1-MCC and G418 from Sigma. DMS-114 cancer cells with homozygous R213X nonsense mutation in the TP53 gene were analyzed for p53 expression using automated capillary electrophoresis western analysis. Untreated cells express no detectable full-length p53, the readthrough product (Fig. 2). Exposure to B1-MCC or G418 caused a similar concentration-dependent increase in full-length p53 (Fig. 2). This result is consistent with the assignment of B1-MCC as G418.

We then compared the activity of B1-TRC and G418. In DMS-114 cells, B1-TRC showed no PTC readthrough activity even at a high concentration of $200 \mu \mathrm{g} / \mathrm{ml}$ (Fig. 3A), whereas G418 did in the same experiment. Similarly, B1-TRC showed no PTC readthrough activity in HDQ-P1 cells, a second cancer cell line with homozygous R213X nonsense mutation in the TP53 gene, while G418 did (Fig. 3B). Additionally, B1-TRC showed no PTC readthrough activity in a cellfree translation assay, whereas G418 did (Fig. 3C). These results indicate that gentamicin B1 has no readthrough activity.

\section{Discussion}

We previously reported that the major components of pharmaceutical gentamicin showed only weak PTC readthrough activity (8), corroborated recently by Friesen (9), but that the minor component B1was a potent readthrough compound (8). Here, we correct the record and show that the B1 used in our study was misidentified by the vendor and was in fact G418, and we provide evidence that synthetic gentamicin B1 is inactive as a readthrough compound. 
Recent X-ray crystallography studies highlight a key role of the 6'-OH in ring 1 of G418 for binding to the ribosomal decoding center and eliciting a conformational change that enables PTC readthrough $(11,12)$. The authors speculate that replacing the 6 '-OH group in ring 1 of G418 with a 6'- $\mathrm{NH}_{2}$ group, as occurs in B1, could preclude ribosomal binding (12). The proposed importance of the 6'-OH group for PTC readthrough is supported by the experimental data we present here comparing G418 with synthetic gentamicin B1, and by a recent study showing that introduction of a $6^{\prime}-\mathrm{NH}_{2}$ group in ring 1 of synthetic pseudotrisaccharides significantly reduces PTC readthrough activity (13). 


\section{References}

1. Mort M, Ivanov D, Cooper DN, Chuzhanova NA (2008) A meta-analysis of nonsense mutations causing human genetic disease. Hum Mutat 29(8):1037-1047.

2. Gentamicin $40 \mathrm{mg} / \mathrm{ml}$ solution for injection or infusion Available at: https://www.medicines.org.uk/emc/product/2394 [Accessed November 9, 2018].

3. Malik V, et al. (2010) Gentamicin-induced readthrough of stop codons in Duchenne muscular dystrophy. Ann Neurol 67(6):771-780.

4. Clancy JP, et al. (2001) Evidence that Systemic Gentamicin Suppresses Premature Stop Mutations in Patients with Cystic Fibrosis. Am J Respir Crit Care Med 163(7):1683-1692.

5. Linde L, Kerem B (2008) Introducing sense into nonsense in treatments of human genetic diseases. Trends Genet 24(11):552-563.

6. Stypulkowska K, Blazewicz A, Fijalek Z, Sarna K (2010) Determination of Gentamicin Sulphate Composition and Related Substances in Pharmaceutical Preparations by LC with Charged Aerosol Detection. Chromatographia 72(11-12):1225-1229.

7. Karpati G, Lochmuller H (2001) When running a stop sign may be a good thing. Ann Neurol 49(6):693-694.

8. Baradaran-Heravi A, et al. (2017) Gentamicin B1: a minor gentamicin component with major nonsense mutation suppression activity. Proc Natl Acad Sci U S A 114(13):34793484.

9. Friesen WJ, et al. (2018) The minor gentamicin complex component, X2, is a potent premature stop codon readthrough molecule with therapeutic potential. PLoS One 13(10):e0206158.

10. Baradaran-Heravi A, et al. (2016) Novel small molecules potentiate premature termination 
codon readthrough by aminoglycosides. Nucleic Acids Res 44(14):6583-98.

11. de Loubresse NG, et al. (2014) Structural basis for the inhibition of the eukaryotic ribosome. Nature 513(7519):517-522.

12. Prokhorova I, et al. (2017) Aminoglycoside interactions and impacts on the eukaryotic ribosome. Proc Natl Acad Sci U S A 114(51):E10899-E10908.

13. Sabbavarapu NM, Pieńko T, Zalman B-H, Trylska J, Baasov T (2018) Exploring eukaryotic versus prokaryotic ribosomal RNA recognition with aminoglycoside derivatives. Medchemcomm 9(3):503-508. 
Table 1. ${ }^{13} \mathrm{C}$ and ${ }^{1} \mathrm{H}$ NMR data comparison for G418 free base, purchased as gentamicin B1 (B1-MCC) and gentamicin B1-acetate salt (B1-TRC) recorded in DMSO- $d_{6}$ and 3:2 DMSO$d_{6} / \mathrm{D}_{2} \mathrm{O}$, respectively, at $600 \mathrm{MHz}$.

\begin{tabular}{|c|c|c|c|c|}
\hline \multirow{2}{*}{ Position } & \multicolumn{2}{|c|}{ G418 free base (B1-MCC) } & \multicolumn{2}{|c|}{$\begin{array}{c}\text { Gentamicin B1-acetate salt (B1- } \\
\text { TRC) }\end{array}$} \\
\hline & ${ }^{13} \mathrm{C}(\delta)$ & ${ }^{1} \mathrm{H}(\delta$, multiplicity $(\mathrm{J} \mathrm{Hz}))$ & ${ }^{13} \mathrm{C}(\delta)$ & ${ }^{1} \mathrm{H}(\delta$, multiplicity $(\mathrm{J} \mathrm{Hz}))$ \\
\hline 1 & 51.7 & 2.59, bt $(9.2)$ & 51.5 & $2.96, \mathrm{~m}$ \\
\hline $\begin{array}{l}2_{\mathrm{ax}} \\
2_{\mathrm{eq}}\end{array}$ & 38.7 & $\begin{array}{c}0.99^{\mathrm{a}} \\
1.74, \mathrm{~m}\end{array}$ & 33.0 & $\begin{array}{l}1.33, \mathrm{~m} \\
2.00, \mathrm{~m}\end{array}$ \\
\hline 3 & 50.3 & $2.51^{a}$ & 49.0 & $3.02, \mathrm{~m}$ \\
\hline 4 & 92.2 & $2.89, \mathrm{t}(9.2)$ & 83.0 & $3.37^{a}$ \\
\hline 5 & 74.2 & $3.34, \mathrm{t}(9.2)$ & 73.9 & $3.49^{a}$ \\
\hline 6 & 86.1 & $2.97, \mathrm{t}(9.2)$ & 86.5 & $3.26, \mathrm{t}(9.1)$ \\
\hline 1 ' & 102.3 & $4.85, \mathrm{~d}(3.1)$ & 97.5 & $5.28, d(3.7)$ \\
\hline 2' & 56.2 & $2.52^{a}$ & 72.4 & 3.36, dd $(9.9,3.7)$ \\
\hline 3' & 74.0 & $3.24, \mathrm{t}(9.2)$ & 73.9 & $3.49^{a}$ \\
\hline $4^{\prime}$ & 72.9 & $3.01^{\mathrm{a}}$ & 71.8 & $3.82^{a}$ \\
\hline 5 & 75.1 & $3.52, \mathrm{bd}(7.8)$ & 70.7 & $3.16, \mathrm{t}(9.6)$ \\
\hline 6' & 66.2 & $3.87^{a}$ & 47.8 & $3.51^{a}$ \\
\hline 7'-Me & 17.4 & $1.01, \mathrm{~d}(6.3)$ & 12.7 & $1.12, \mathrm{~d}(7.0)$ \\
\hline $1 "$ & 100.3 & $4.92, \mathrm{~d}(3.2)$ & 101.6 & $4.88, \mathrm{~d}(3.6)$ \\
\hline 2"' & 69.7 & $3.43, \mathrm{dd}(10.2,3.2)$ & 67.9 & $3.93, \mathrm{dd}(10.8,3.6)$ \\
\hline 3" & 64.2 & $2.28, \mathrm{~d}(10.2)$ & 65.0 & $3.07, \mathrm{~d}(10.8)$ \\
\hline 4"' & 71.2 & 1 & 70.9 & 1 \\
\hline $\begin{array}{l}5 "{ }^{\prime \prime a x} \\
5 "{ }_{\text {eq }}\end{array}$ & 67.4 & $\begin{array}{l}3.89, d(11.9) \\
3.05, d(11.9)\end{array}$ & 68.4 & $\begin{array}{c}3.82^{\mathrm{a}} \\
3.21, \mathrm{~d}(12.5)\end{array}$ \\
\hline 3"'-NH-Me & 38.1 & $2.45 \mathrm{~s}$ & 36.2 & $2.66, \mathrm{~s}$ \\
\hline 4"-Me & 23.9 & $0.97, \mathrm{~s}$ & 22.7 & $1.13, \mathrm{~s}$ \\
\hline
\end{tabular}

${ }^{a}$ Multiplicity not determined due to overlapping signals - chemical shifts determined from 2D NMR data. 


\section{Figures and legends}

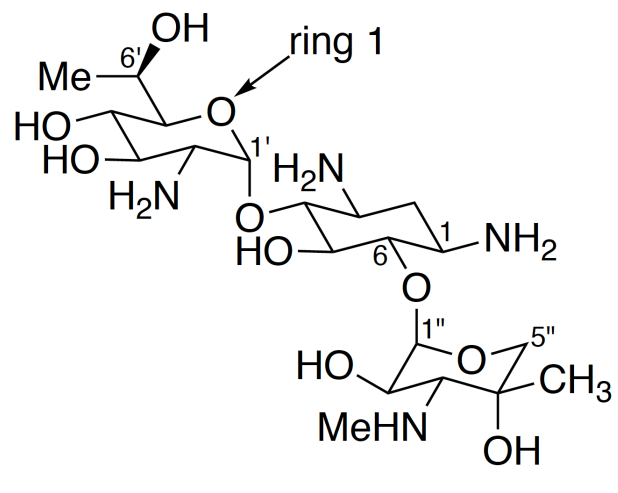

G418 free base

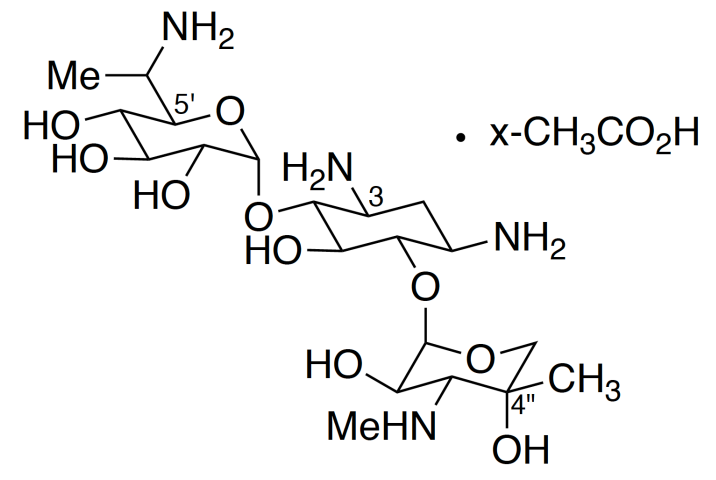

Gentamicin B1-acetate salt

Figure 1. Structure of G418 and gentamicin B1 assigned from NMR data in Table 1. 


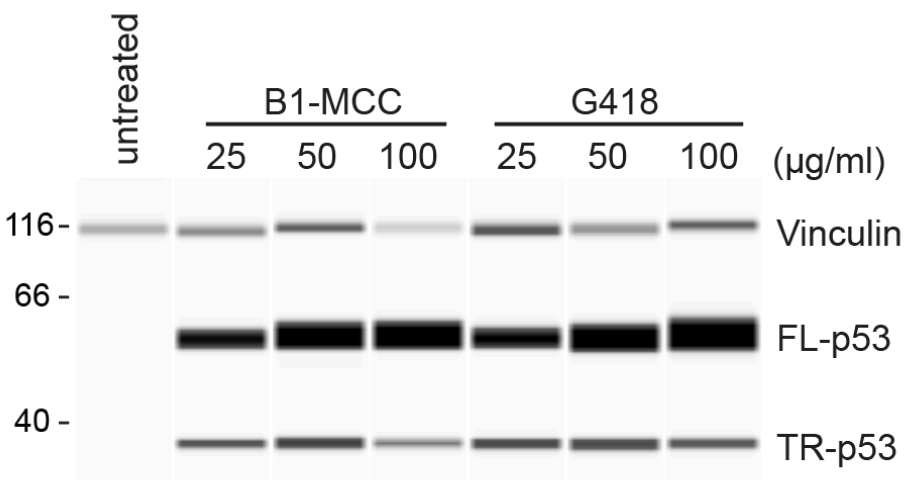

Figure 2. Comparison of the PTC readthrough activity of B1-MCC and G418. DMS-114 cells were incubated with the indicated concentrations of B1-MCC sulfate and G418 sulfate for $72 \mathrm{~h}$ and the production of full-length p53 (FL-p53) and truncated p53 (TR-p53) was determined using automated capillary electrophoresis western analysis with vinculin as a protein loading control. Molecular weight markers are shown on the left in kDa. 
A

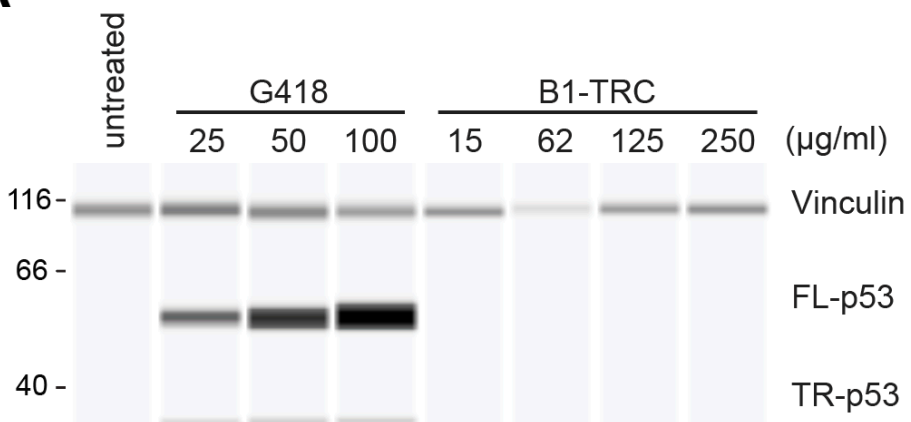

B

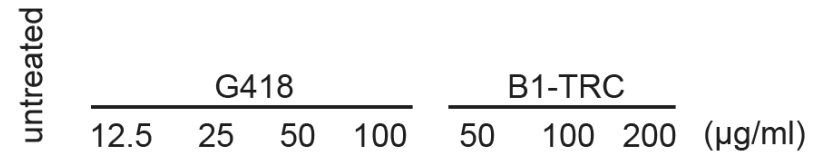$$
116-0=0=0
$$

66 -

$40-$
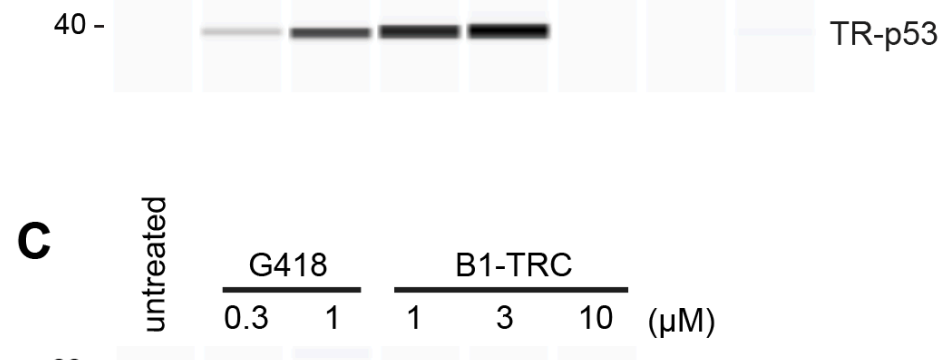

$66-$

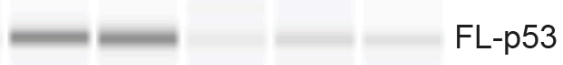

$40-$ TR-p53

FL-p53 $11 \quad 2.2 \quad 2.2 \quad 0.9 \quad 1.1 \quad 1$

Figure 3. Effect of gentamicin B1 on PTC readthrough in cells and in a cell-free assay. DMS-114 cells (A) and HDQ-P1 cells (B) were incubated with the indicated concentrations of G418 sulfate or gentamicin B1 acetate for $48 \mathrm{~h}$ (A) or $72 \mathrm{~h}$ (B) and production of full-length p53 (FL-p53) and truncated p53 (TR-p53) was determined using automated capillary electrophoresis western analysis. Vinculin was used as a protein loading control. (C) Synthetic R213X TP53 mRNA was subjected to in vitro translation in the presence of the indicated concentrations of G418 sulfate or 
bioRxiv preprint doi: https://doi.org/10.1101/476564; this version posted November 23, 2018. The copyright holder for this preprint (which was not certified by peer review) is the author/funder, who has granted bioRxiv a license to display the preprint in perpetuity. It is made available under aCC-BY-NC-ND 4.0 International license.

gentamicin B1 acetate for 30 min and p53 was detected as in A and B. FL-p53 signal intensity relative to untreated control is shown below the lanes. 